\title{
Utility of venous compression in deep venous thrombosis evaluation revisited
}

\author{
Kocakoc E1, Bhatt S², Dogra VS² \\ Department of Radiology, Faculty of Medicine, Bezmialem Vakif University, Istanbul, Turkey. \\ Vikram_Dogra@URMC.rochester.edu
}

\begin{abstract}
Objective: Ultrasound venous compression (UVC) is considered the gold standard for confirmation of deep venous thrombosis (DVT) of the lower extremities. The objective of this study was to assess the contribution and significance of venous compression in comparison to color flow duplex (CFD) ultrasonography alone in the diagnosis of DVT.

Methods: Retrospective analysis was performed of all DVT studies during two years period. DVT examinations were performed with a 5.8-7.6 MHz linear broadband transducer following the American Institute of Ultrasound in Medicine guidelines for the performance of DVT examination. The images were categorized as normal, partial thrombus, or complete thrombus.

Results: A total of 428 patients comprised the study group. In total, 467 DVT examinations were performed (39 patients had bilateral examinations). Of the lower extremity examinations, 347/467were normal and 120/467 were abnormal. Complete thrombus was evident in 49/120 patients, while 71/120 patients had partial thrombus. We observed the thrombus on gray scale imaging in all 120 positive patients. No patient had venous compression negative for thrombus and CFD positive for thrombus. There was one patient with visualization of thrombus on gray scale imaging and complete venous compression (negative for thrombus). CFD examination of this patient was also negative for thrombus.

Conclusion: UVC did not provide any additional information for the diagnosis of DVT. If CFD demonstrates the presence of DVT, venous compression is not necessary, although it can further confirm the presence of DVT. No additional DVTs were diagnosed by using venous compression alone (Tab. 2, Fig. 2, Ref. 27). Full Text in PDF www.elis.sk.

Key words: deep venous thrombosis, Color flow, Doppler, venous compression ultrasonography, ultrasound, veins, DVT.
\end{abstract}

Deep venous thrombosis (DVT) is a common and life-threatening clinical condition with an average incidence of approximately 70 per 100,000 patients per year (1). Clinical diagnosis of DVT is not reliable, so imaging is necessary to confirm its presence. Traditionally, contrast venography (CV) was considered the gold standard technique for diagnosis of DVT, but it has some drawbacks such as contrast-induced nephropathy, allergy, procedurerelated pain, superficial thrombophlebitis, and radiation exposure. In addition, post-procedure DVT and skin necrosis may be observed in about $8 \%$ of examinations in spite of using non-ionic contrast media. About $5 \%$ of $\mathrm{CV}$ examinations are inadequate and non-diagnostic $(2,3)$. Because of these limitations, alternative non-invasive diagnostic tests are used to evaluate DVT. Several serologic markers of thrombosis have been investigated, and the D-dimer blood test has emerged as the most useful. The D-dimer test reveals a breakdown product of the cross-linked fibrin clot (4).

${ }^{1}$ Department of Radiology, Faculty of Medicine, Bezmialem Vakif University, Istanbul, Turkey, and ${ }^{2}$ Department of Imaging Sciences, University of Rochester School of Medicine, Rochester, NY 14642

Address for correspondence: V.S. Dogra, MD, University of Rochester School of Medicine and Dentistry, Rochester, NY 14642

Phone: +585.273.4388
Positive test has a very low predictive value, but negative test has a reported negative predictive value of more than $97 \%$ (5). Magnetic resonance (MR) venography (6) and computed tomography (CT) venography (7) are also new and evolving techniques for the evaluation of DVT, but these techniques are expensive, require post-processing, and more time to perform the examination.

Currently, ultrasound (US) using venous compression is considered the gold standard for confirmation of DVT $(5,8,9)$. This examination is inexpensive and easy to perform.

The objective of this study is to assess the contribution and significance of ultrasound venous compression (UVC) compared to color flow Doppler (CFD) alone in the diagnosis of DVT. We also aim to assess the number of patients diagnosed with DVT by venous compression alone.

\section{Methods}

We performed a retrospective analysis of DVT studies during two-year period. All patients with diagnostic quality images were included in the study. If a patient had more than one examination, the follow-up examinations were not included in this retrospective analysis. DVT examinations were performed using a 5.8-7.6 MHz (PLT604AT) linear broadband transducer on a Toshiba Aplio 
Tab. 1. Right/left lower extremity distribution of DVT examinations.

\begin{tabular}{lrcc}
\hline & Total & $\begin{array}{c}\text { Right lower } \\
\text { extremity }\end{array}$ & $\begin{array}{c}\text { Left lower } \\
\text { extremity }\end{array}$ \\
\hline Normal Examinations & 347 & 164 & 183 \\
Complete, DVT & 49 & 29 & 20 \\
Partial, DVT & 71 & 44 & 27 \\
Total \# of examinations & 467 & 237 & 230 \\
\hline
\end{tabular}

Tab. 2. Associated findings observed with DVT examinations.

\begin{tabular}{lcc}
\hline Associated findings & Number & Percentage (\%) \\
\hline Baker's cyst & 24 & 40 \\
Lymph nodes & 12 & 20 \\
Soft tissue edema & 9 & 15 \\
Duplicate veins & 5 & 8.33 \\
Venous insufficiency & 5 & 8.33 \\
Soft tissue masses & 3 & 5 \\
Thrombophlebitis & 2 & 3.33 \\
\hline Total & 60 & 100 \\
\hline
\end{tabular}

machine (Toshiba; Tokyo, Japan), following the AIUM guidelines. The common femoral vein (CFV), femoral vein (FV) and popliteal vein (PV) were evaluated in all patients. The examinations were performed with patients lying in a supine position with the head slightly elevated (about 20-30 degrees). The PV was evaluated with the patient's knee flexed in external rotation in a supine position or in a prone position. Calf veins were not routinely evaluated and if evaluated, results were not included in this study. A representative image of CFV from the opposite extremity was also obtained. Venous segments were examined continuously in B-mode; compressibility of these veins was assessed at $2-3 \mathrm{~cm}$ intervals in the axial plane. CFD sonography with pulsed Doppler and augmentation maneuver was used to document venous flow. CFD examination was performed mainly in longitudinal plane. The color scale was set to lower values to detect slow flow, but if the color signals were observed in the
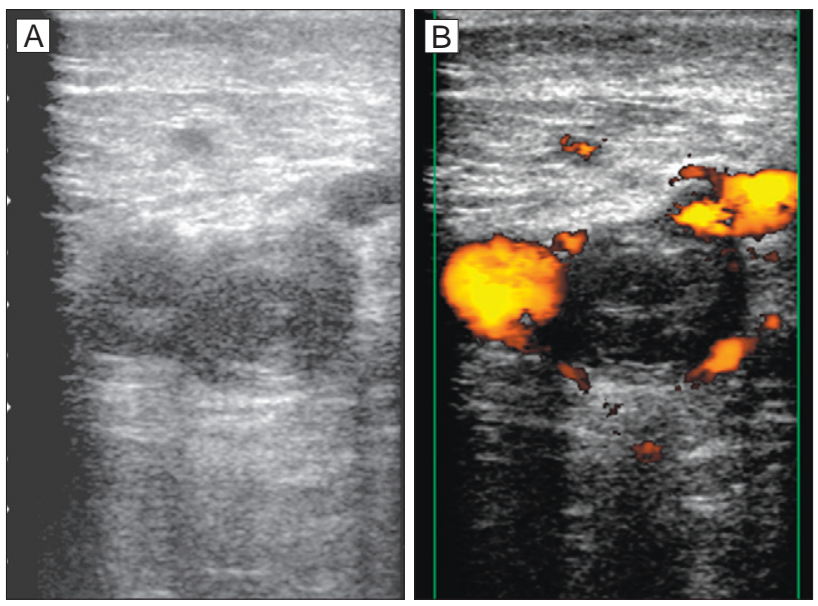

Fig. 1. (A) Gray scale US image of common femoral vein (CFV) demonstrates an intraluminal filling defect resulting in enlargement of the vein.(B)Corresponding power Doppler confirms the diagnosis of complete thrombus involving the CFV.

soft tissues, it was considered over-saturation and the color scale sets were increased to reduce these artifacts. Soft tissues were also assessed for alternative diagnosis, such as Baker's cyst or hematoma, especially in the popliteal region. Two radiologists, blinded to the initial results, independently reviewed the images. Examination findings were categorized as normal, partial thrombus, or complete thrombus. If venous segments were fully compressible and blood flow was seen within the veins on CFD evaluation, examination was categorized as normal. If venous segments were non-compressible or blood flow was not present within the venous lumen on CFD evaluation, examination was categorized as a complete thrombus. If venous segments were partially compressible or intraluminal filling defect was seen in the vein on CFD, examination was categorized as partial throm-
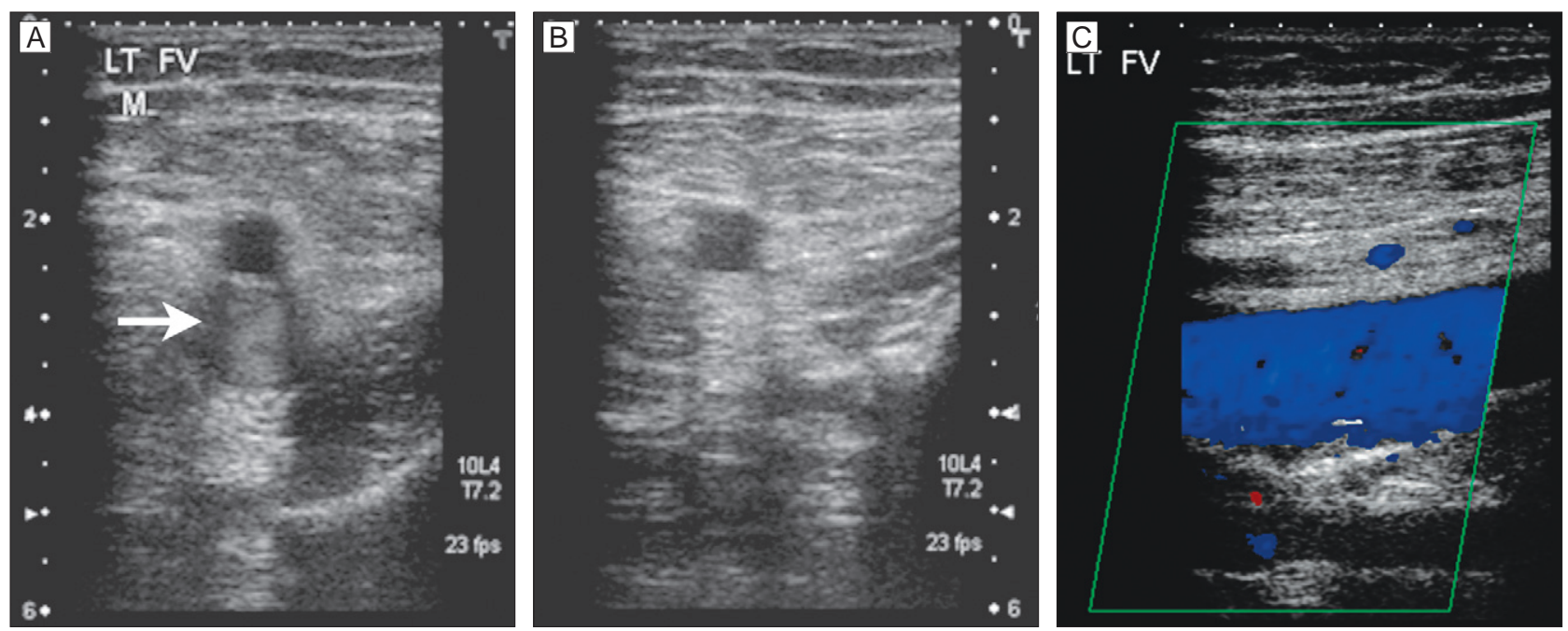

Fig. 2. (A) Gray scale US image without venous compression demonstrates an isoechoic intraluminal filling defect involving the left femoral vein (FV), suggesting a thrombus (arrow) (B)Ultrasound venous compression reveals complete compressibility of the FV. (C) Color flow Doppler also demonstrates a normal flow within the FV. 
bus. If thrombus was observed only in the superficial veins, superficial thrombophlebitis was diagnosed.

\section{Results}

The study group comprised of 428 patients. There were 135 males (with a mean age of 56.3 years) and 293 females (with a mean age of 57.1 years). In total, 467 DVT exams were performed (39 patients had bilateral lower extremity exams). We performed 198 exams on the right side, and 191 on the left side only. Of the 467, 347 lower extremity examinations were normal (74 \%). In total, 120 of 467 (26\%) exams were abnormal. Forty-nine patients out of 120 (40.8\%) had complete thrombus. Seventy-one patients out of 120 (59.2 \%) had partial thrombus (Tab. 1). DVT was more common in the right lower extremity (60.8 \% of patients). The femoral vein was the most commonly involved vein (49/120; 40.8 $\%)$ followed by the popliteal vein (37/120; $30.8 \%)$, and the common femoral vein (34/120; $28.4 \%$ ) (Figs 1 and 2).

We observed the thrombus on gray scale imaging in all 120 patients with DVT. No patient had absence of venous CFD (positive for thrombus) with negative venous compression (negative for thrombus). There was one patient with visualization of thrombus on gray scale imaging and complete venous compression (negative for thrombus). CFD examination of this patient was also negative for thrombus (Fig. 2). Sixty lower extremity examinations (12.8 \%) for DVT also had associated additional findings; Baker's cyst was the most common (24/60). Findings observed during examinations are summarized in Table 2.

\section{Discussion}

Lower extremity DVT is a very common and serious clinical condition requiring early diagnosis and treatment before lifethreatening complications such as pulmonary embolism occur (10). DVT cannot be reliably diagnosed by clinical examination alone because the signs and symptoms (e.g., leg swelling, pain, etc.) are many and nonspecific (2). It has been reported that only $40 \%$ of patients with DVT have true clinical signs of the disorder, and false-positive clinical signs are observed in about $50 \%$ of patients (11). Because of the inaccuracy of clinical examination and severe consequences of erroneous diagnosis, imaging is necessary for diagnosing DVT $(8,11)$.

Although CV had been considered the gold standard technique for the diagnosis of DVT, it is no longer performed because of its invasive nature, radiation exposure, discomfort to the patient, and side effects from radiographic contrast agents (2).

UVC has effectively replaced CV for the assessment of patients with suspected DVT (12). The main criteria for the diagnosis of DVT is lack of compression of the vein, the presence of intraluminal echogenic material on gray scale examination, the filling defect on CFD examination, and the absence of spontaneous or forced blood flow (13). The other criteria are increased venous diameter, venous wall changes, and collateral veins with increased flow $(13,14)$. Gray scale compression US assesses the presence of thrombus, whereas CFD demonstrates the presence of the blood flow within the vein (15). Gray scale visualization of intraluminal thrombus is considered unreliable for the diagnoses of DVT (16); however, our experience has been to the contrary. In our study, we were able to see the intraluminal filling defect on gray scale ultrasound in all 120 DVT positive patients. We concluded that improvements in US technology could have made the original criteria for the diagnosis of DVT obsolete, leading to the difference in the results we observed.

A normal deep vein should fill completely on CFD examination and a DVT will appear as a filling defect inside the vein lumen (8). Visualization of the color flow outside the vessel wall is a common artifact. One important disadvantage of CFD is oversaturation of color; it may obscure partial intraluminal occlusions. Color gain and other sets should be adjusted to minimize this in order to permit proper evaluation of mural thrombus. CFD is very useful for the rapid detection of deep veins and immediate assessment of their patency $(13,17,18)$. CFD is a useful technique in special situations in which the compression test has limitations. CFD evaluation is useful in patients after surgery, and those with burns, large thighs, and swollen calves in the region of the inguinal ligament and the adductor canal $(4,8,13)$. CFD may allow the detection of spontaneous flow in small calf veins. In addition, CFD imaging results in a shorter examination time and faster evaluation of ancillary pathologic conditions $(8,16,19)$. CFD examination alone without venous compression has shown excellent correlation with venography for evaluation of DVT with $95 \%$ sensitivity and 99 $\%$ specificity, $95 \%$ positive predictive value and $99 \%$ negative predictive value $(18,20)$.

An important advantage of CFD is that it can be used to evaluate the leg for other possible causes of symptoms besides DVT. US can demonstrate pseudoaneurysms, enlarged lymph nodes, cellulitis, saphenous vein clot, and Baker's cysts. Alternate diagnoses that explain patients' symptomatology are seen in approximately $10 \%$ of patients undergoing DVT evaluation (21). In the present study approximately $12 \%$ of patients evaluated for DVT had alternate diagnoses explaining their DVT symptoms.

A recent meta analysis of the diagnostic accuracy of ultrasonography for DVT showed that while UVC only has $93.8 \%$ pooled sensitivity and $97.8 \%$ specificity, CFD has $95.8 \%$ pooled sensitivity and $92.7 \%$ specificity for proximal DVT (22), suggesting that results of CFD examination are comparable to those of UVC.

UVC is very easy and simple technique that allows quick examination of limbs. However, this test may be falsely positive because of focal vein incompressibility due to anatomic variants at specific sites, including the femoral vein at the level of the saphenous vein opening, the deep femoral vein, and the poplitealfemoral vein junction at the level of the adductor hiatus. In such cases, CFD is useful to show complete vascular filling.

UVC has the potential to break off the femoral vein thrombus and cause pulmonary emboli (PE) (9). Although rare, compressionrelated fatal $\mathrm{PE}$ has been reported in the literature (23). Therefore, if CFD is apparently demonstrating DVT, no additional compression should be performed.

During the initial stages of the development of US for evaluation of DVT, the main challenge was to image the acute thrombus $(24,25)$. The technology that existed approximately 20 years 
ago was not capable of doing so, therefore, indirect means of diagnosing a thrombus within a vein were adopted (21, 24-27). Today, improvements in ultrasound technology, CFD, and power Doppler technology enable us to detect slow flow and venous intraluminal filling defects with confidence. CFD examination with current high-end machines can depict the intraluminal filling defects with near certainty, as in our study where all the patients with DVT could be identified with CFD and no patient was diagnosed with DVT by UVC alone. In view of the new developments in US technology, we suggest that UVC should only be performed in the presence of intraluminal filling defect detected on CFD or power Doppler examination.

Our study has limitations, as it is retrospective and involves a small number of patients. Our results would need to be additionally confirmed by a larger prospective study. Although we did not perform routinely bilateral examination in emergency cases; it would be good to perform both lower limbs examination. Bilateral examination allows not only detect asymptomatic DVT but also compare both limbs for anatomic variations.

In conclusion, ultrasound venous compression did not provide any additional information for the diagnosis of DVT. If CFD demonstrates the presence of DVT, ultrasound venous compression is not necessary, although it can further confirm the presence of DVT.

\section{References}

1. Anderson FA, Wheeler HB, Goldberg RJ et al. A population-based perspective of the hospital incidence and case-fatality rates of deep vein thrombosis and pulmonary embolism: the Worcester DVT study. Ann Intern Med 1991; 151: 933-938.

2. Redman HC. Deep venous thrombosis: is contrast venography still the diagnostic "gold standard"? Radiology 1988; 168: 227-228.

3. Bettmann MA, Robbins A, Braun SD, Wetzner S, Dunnick NR, Finkelstein J. Contrast venography of the leg: diagnostic efficacy, tolerance, and complication rates with ionic and nonionic contrast media. Radiology 1987; 165: 113-116.

4. Fraser JD, Anderson DR. Venous protocols, techniques, and interpretations of the upper and lower extremities. Radiol Clin N Am 2004; 42: 279-296.

5. Tracy JA, Edlow JA. Ultrasound diagnosis of deep venous thrombosis. Emerg Med Clin N Am 2004; 22: 775-796.

6. Laissy JP, Cinqualbre A, Loshkajian A et al. Assessment of deep venous thrombosis in the lower limbs and pelvis: MR venography versus duplex Doppler sonography. AJR Am J Roentgenol 1996; 167: 971-975.

7. Loud PA, Katz DS, Bruce DA, Klippenstein DL, Grossman ZD. Deep venous thrombosis with suspected pulmonary embolism: detection with combined CT venography and pulmonary angiography. Radiology 2001; 219: 498-502.

8. Cronan JJ. Venous thromboembolic disease: the role of US. Radiology 1993; 186: 619-630.

9. Cronan JJ. Controversies in venous ultrasound. Semin Ultrasound CT MR 1997; 18: 33-38.
10. Rose SC, Zwiebel WJ, Nelson BD et al. Symptomatic lower extremity deep venous thrombosis: accuracy, limitations, and role of color duplex flow imaging in diagnosis. Radiology 1990; 175: 639-644.

11. Greenfield LJ. Venous thromboembolic disease. In: Moore WS (ed). Vascular surgery: a comprehensive review. Philadelphia, PA: WB Saunders, 1991: 669-679.

12. Rosen MP, McArdle C. Controversies in the use of lower extremity sonography in the diagnosis of acute deep vein thrombosis and a proposal for a unified approach. Semin Ultrasound CT MR 1997; 18: 362-368.

13. Dauzat M, Laroche JP, Deklunder G et al. Diagnosis of acute lower limb deep venous thrombosis with ultrasound: trends and controversies. J Clin Ultrasound 1997; 25: 343-358.

14. Hertzberg BS, Kliewer MA, DeLong DM et al. Sonographic assessment of lower limb vein diameters: implications for the diagnosis and characterization of deep venous thrombosis. AJR Am J Roentgenol 1997; 1253-1257.

15. Theodoro D, Blaivas M, Duggal S, Snyder G, Lucas M. Real-time $\mathrm{B}$-mode ultrasound in the ED saves time in the diagnosis of deep vein thrombosis (DVT). Am J Emerg Med 2004; 22: 197-200.

16. Machi J, Sigel B, Beitler JC, Coelho JC, Justin JR. Relation of in vivo blood flow to ultrasound echogenicity. J Clin Ultrasound 1983; 11:3-10.

17. Lewis BD, James EM, Welch TJ, Joyce JW, Hallett JW, Weaver AL. Diagnosis of acute deep venous thrombosis of the lower extremities: prospective evaluation of CFD flow imaging versus venography. Radiology 1994; 192: 651-655.

18. Polak JF, Culter SS, O’Leary DH. Deep veins of the calf: assessment with CFD flow imaging. Radiology 1989; 171: 481-485.

19. Foley WD, Middleton WD, Lawson TL, Erickson S, Quiroz FA, Macrander S. CFD ultrasound imaging of lower-extremity venous disease. AJR Am J Roentgenol 1989; 152: 371-376.

20. Glajchen N, Shapiro RS. Underassessment of lower extremity deep venous thrombosis using CFD compared to compression sonography. Comput Med Imaging Graph 1997; 21: 331-335.

21. Cronan JJ, Dorfman GS, Grusmark J. Lower-extremity deep venous thrombosis: further experience with and refinements of US assessment. Radiology 1988; 168: 101-107.

22. Goodacre S, Sampson F, Thomas S, van Beek E, Sutton A. Systematic review and meta-analysis of the diagnostic accuracy of ultrasonography for deep vein thrombosis. BMC Med Imaging 2005; 5: 6.

23. Perlin SJ. Pulmonary embolism during compression US of the lower extremity. Radiology 1992; 184: 165-166.

24. Talbot SR. Use of real-time imaging in identifying deep venous obstruction: a preliminary report. Bruit 1982; 7: 41-42.

25. Raghavendra BN, Rosen RJ, Lam S, Riles T, Horii SC. Deep venous thrombosis: detection by high-resolution real-time ultrasonography. Radiology 1984; 152: 789-793.

26. Raghavendra BN, Horii SC, Hilton S, Subramanyam BR, Rosen RJ, Lam S. Deep venous thrombosis: detection by probe compression of veins. J Ultrasound Med 1986; 5: 89-95.

27. Appelman PT, De Jong TE, Lampmann LE. Deep venous thrombosis of the leg: US findings. Radiology 1987; 163: 743-746. 